\title{
Kragujevac Trees and Their Energy
}

\section{Ivan Gutman}

\section{INVITED PAPER}

\begin{abstract}
The Kragujevac trees with a fixed degree and a fixed order, with maximal and minimal energy are determined.
\end{abstract}

Keywords: energy (of graph), spectrum (of graph), tree, Kragujevac tree.

\section{Introduction: Kragujevac trees}

The atom-bond connectivity $(A B C)$ index is one of the currently much studied molecular structure-descriptors based on the degrees of the vertices of the molecular graph $[7,11]$. The theory of the $A B C$ index is irrelevant for the considerations in the present paper; for its details see $[6,17]$

Within recent researches $[1,2,5,8,12-14,20,22]$ aimed at the characterization of trees that have minimal $A B C$ index, a class of trees has been encountered for which the name "Kragujevac trees" was proposed [20].

A connected acyclic graph is called a tree. The number of vertices of a tree $T$ is its order, denoted by $n(T)$. A rooted tree is a tree in which one particular vertex is distinguished; this vertex is referred to as the root (of the rooted tree) [18].

In order to define the Kragujevac trees, we first explain the structure of its branches.

Definition 1. Let $P_{3}$ be the 3-vertex tree, rooted at one of its terminal vertices, see Fig. 1 . For $k=2,3, \ldots$, construct the rooted tree $B_{k}$ by identifying the roots of $k$ copies of $P_{3}$. The vertex obtained by identifying the roots of $P_{3}$-trees is the root of $B_{k}$.

Examples illustrating the structure of the rooted tree $B_{k}$ are depicted in Fig. 1.

Manuscript received April 23, 2014; accepted May 27, 2014.

Ivan Gutman is with the Faculty of Science, University of Kragujevac, Serbia 

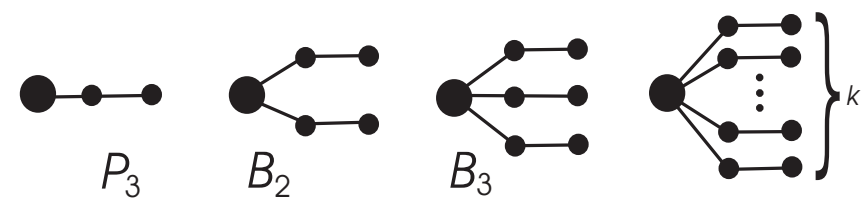

$B_{k}$

Fig. 1. The rooted trees $B_{2}, B_{3}$, and $B_{k}$, obtained, respectively, by identifying the roots of 2,3 , and $k$ copies of $P_{3}$. Their roots are indicated by large dots.

Definition 2. Let $d \geq 2$ be an integer. Let $\beta_{1}, \beta_{2}, \ldots, \beta_{d}$ be rooted trees specified in Definition 1, i.e., $\beta_{1}, \beta_{2}, \ldots, \beta_{d} \in\left\{B_{2}, B_{3}, \ldots\right\}$. A Kragujevac tree $T$ is a tree possessing a vertex of degree $d$, adjacent to the roots of $\beta_{1}, \beta_{2}, \ldots, \beta_{d}$. This vertex is said to be the central vertex of $T$, whereas $d$ is the degree of $T$. The subgraphs $\beta_{1}, \beta_{2}, \ldots, \beta_{d}$ are the branches of $T$. Recall that some (or all) branches of $T$ may be mutually isomorphic.

A typical Kragujevac tree is depicted in Fig. 2.

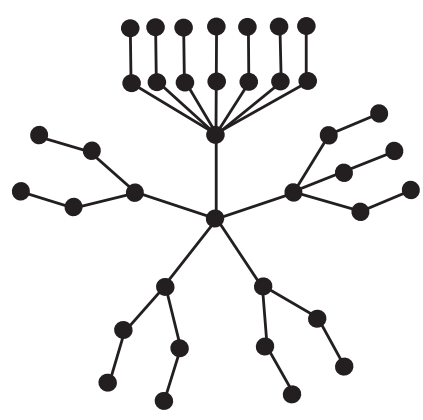

Fig. 2. A Kragujevac tree of degree $d=5$, in which $\beta_{1}, \beta_{2}, \beta_{3} \cong B_{2}, \beta_{4} \cong B_{3}$, and $\beta_{5} \cong B_{7}$.

The branch $B_{k}$ has $2 k+1$ vertices. Therefore, if in the Kragujevac tree $T$, specified in Definition $2, \beta_{i} \cong B_{k_{i}}, i=1,2, \ldots, d$, then its order is

$$
n(T)=1+\sum_{i=1}^{d}\left(2 k_{i}+1\right) .
$$

Evidently, if the degree $d$ of a Kragujevac tree is even (resp. odd), then its order $n$ is odd (resp. even).

It is now easy to verify that the order of a Kragujevac tree must be least $n=11$, and that there exist Kragujevac trees of order $n$, for all $n \geq 11$, except $n=12$ and $n=14$. This fact is illustrated by the examples depicted in Fig. 3. 


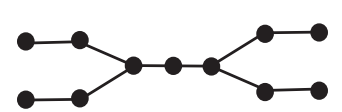

$d=2$

$n=11$

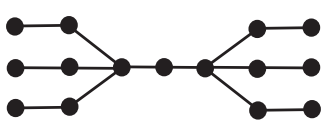

$d=2$

$n=15$

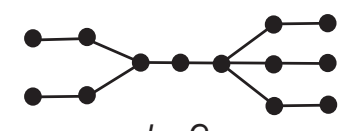

$d=2$

$n=13$

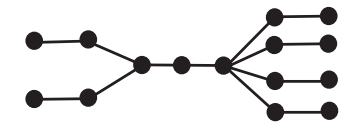

$d=3$

$n=15$

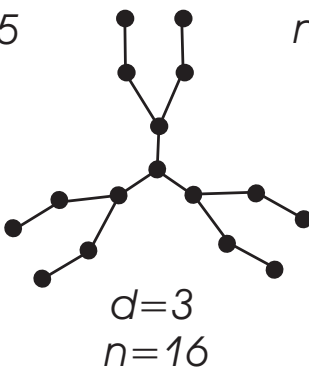

Fig. 3. The smallest Kragujevac trees.

\section{Introduction: Graph energy}

Let $G$ be a simple graph and let its vertices be $v_{1}, v_{2}, \ldots, v_{n}$. Then the adjacency matrix of $G$ is the square matrix $\mathbf{A}(G)$ of order $n$, whose $(i, j)$-entry is defined as [18]

$$
a_{i j}= \begin{cases}0 & \text { if } i=j \\ 1 & \text { if the vertices } v_{i} \text { and } v_{j} \text { are adjacent } \\ 0 & \text { if the vertices } v_{i} \text { and } v_{j} \text { are not adjacent. }\end{cases}
$$

The eigenvalues $\lambda_{1}, \lambda_{2}, \ldots, \lambda_{n}$ of $\mathbf{A}(G)$ form the spectrum of the graph $G$. Details of the theory of graph spectra can be found in the books $[3,4]$. The energy of the graph $G$ is a spectrum-based graph invariant, defined as

$$
E=E(G)=\sum_{i=1}^{n}\left|\lambda_{i}\right|
$$

In the recent years, graph energy is being extensively studied, and new papers on this matter appear in the mathematical literature almost every week. Details of the theory of graph energy can be found in the reviews $[10,16]$ and the monograph [21]. In the present 
work, we offer a further contribution to the theory of graph energy, by characterizing the Kragujevac trees with minimal and maximal $E$-values.

In what follows, we outline a few results of spectral graph theory and the theory of graph energy that will be needed in the subsequent considerations.

The characteristic polynomial of the graph $G$, denoted by $\phi(G, \lambda)$, is defined as the characteristic polynomial of the adjacency matrix $\mathbf{A}(G)$, i.e.,

$$
\phi(G, \lambda)=\operatorname{det}\left[\lambda \mathbf{I}_{n}-\mathbf{A}(G)\right]
$$

where $\mathbf{I}_{n}$ is the unit matrix of order $n[3,4]$.

If the graph $G$ consists of (disconnected) components $H_{1}, H_{2}, \ldots, H_{p}$ then

$$
\phi(G, \lambda)=\prod_{k=1}^{p} \phi\left(H_{k}, \lambda\right) .
$$

For $k \geq 2$, a $k$-matching of a graph $G$ is a selection of its $k$ independent (= mutually non-touching) edges. The number of distinct $k$-matchings of $G$ is denoted by $m(G, k)$. It is both consistent and convenient to define $m(G, 0)=1$ for all graphs $G$, and $m(G, 1)=$ number of edges of $G$.

If the graph $G$ is acyclic (which, of course, includes the case when $G$ is a tree), then $[3,4,19]$

$$
\phi(G, \lambda)=\sum_{k \geq 0}(-1)^{k} m(G, k) \lambda^{n-2 k} .
$$

If $k$ is sufficiently large, then $m(G, k)=0$. In particular, $m(G, k)=0$ whenever $k>n / 2$. Therefore, the expression on the right-hand side of Eq. (2) is a monic polynomial of degree $n$.

If Eq. (2) holds, then the energy of the graph $G$ satisfies the integral expression [9]

$$
E(G)=\frac{2}{\pi} \int_{0}^{+\infty} \frac{1}{x^{2}} \ln \left[\sum_{k \geq 0} m(G, k) x^{2 k}\right] d x .
$$

A remarkable consequence of formula (3) is [9]:

Lemma 3. Let $G_{1}$ and $G_{2}$ be two graphs whose characteristic polynomials satisfy Eq. (2). If $m\left(G_{1}, k\right) \geq m\left(G_{2}, k\right)$ holds for all $k \geq 1$, then $E\left(G_{1}\right) \geq E\left(G_{2}\right)$. If, in addition, $m\left(G_{1}, k\right)>m\left(G_{2}, k\right)$ holds for at least one value of $k$, then $E\left(G_{1}\right)>E\left(G_{2}\right)$.

Let $e$ be an edge of the graph $G$, connecting the vertices $u$ and $v$. Then $m(G, k)=$ $m(G-e, k)+m(G-u-v, k-1)$, which combined with Eq. (2) yields [9, 19]

$$
\phi(G, \lambda)=\phi(G-e, \lambda)-\phi(G-u-v, \lambda) .
$$


In what follows we shall need a special case of Eq. (4).

Let $v$ be a vertex of the graph $G$, adjacent to the vertices $u_{1}, u_{2}, \ldots, u_{d}$. Let for $i=$ $1,2, \ldots, d, e_{i}$ be the edge connecting $v$ and $u_{i}$. Then a $k$-fold application of formula (4) results in $[9,19]$

$$
\phi(G, \lambda)=\phi\left(G-e_{1}-e_{2}-\cdots-e_{d}, \lambda\right)-\sum_{i=1}^{d} \phi\left(G-u_{i}-v, \lambda\right) .
$$

We conclude this section by computing the characteristic polynomial of the rooted tree $B_{k}$ from Definition 1. Let $v$ be its root, and $u_{1}, u_{2}, \ldots, u_{k}$ its first neighbors. Let for $i=$ $1,2, \ldots, k$, the edge connecting $v$ and $u_{i}$ be denoted by $e_{i}$. Then

(a) $B_{k}-e_{1}-e_{2}-\cdots-e_{k}$ consists of an isolated vertex $P_{1}$ and $k$ copies of 2-vertex trees $P_{2}$

(b) For any $i=1,2, \ldots, k, B_{k}-u_{i}-v$ consists of an isolated vertex $P_{1}$ and $k-1$ copies of $P_{2}$.

Knowing that $\phi\left(P_{1}, \lambda\right)=\lambda$ and $\phi\left(P_{2}, \lambda\right)=\lambda^{2}-1$, by applying Eq. (1) we get

$$
\begin{aligned}
\phi\left(B_{k}-e_{1}-e_{2}-\cdots-e_{k}, \lambda\right) & =\lambda\left(\lambda^{2}-1\right)^{k} \\
\phi\left(B_{k}-u_{i}-v, \lambda\right) & =\lambda\left(\lambda^{2}-1\right)^{k-1}
\end{aligned}
$$

which bearing in mind Eq. (5) yields

$$
\phi\left(B_{k}, \lambda\right)=\lambda\left(\lambda^{2}-1\right)^{k}-k \lambda\left(\lambda^{2}-1\right)^{k-1}
$$

and finally

$$
\phi\left(B_{k}, \lambda\right)=\lambda\left(\lambda^{2}-1\right)^{k-1}\left(\lambda^{2}-k-1\right)
$$

\section{An auxiliary result}

Let $k$ and $h$ be integers, $k-2 \geq h \geq 2$. Let $T_{0}$ be a tree and $v$ its vertex. Construct the tree $X$ be attaching to the vertex $v$ of $T_{0}$ two new branches, by connecting $v$ with the root vertices of $B_{k}$ and $B_{h}$, see Fig. 4. Construct the tree $Y$ by attaching to the vertex $v$ of $T_{0}$ two new branches, by connecting $v$ with the root vertices of $B_{k-1}$ and $B_{h+1}$, see Fig. 4 . Both trees $X$ and $Y$ possess $n\left(T_{0}\right)+2(k+h+1)$ vertices. 


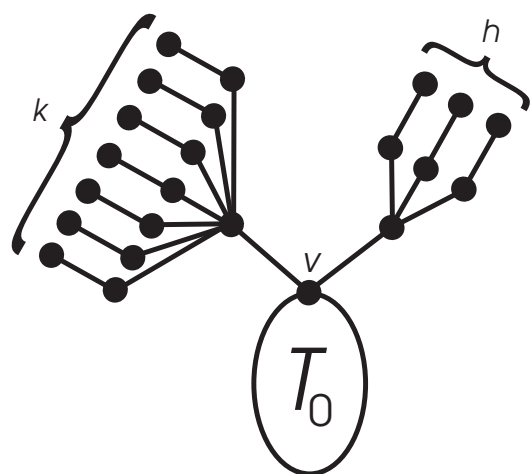

X

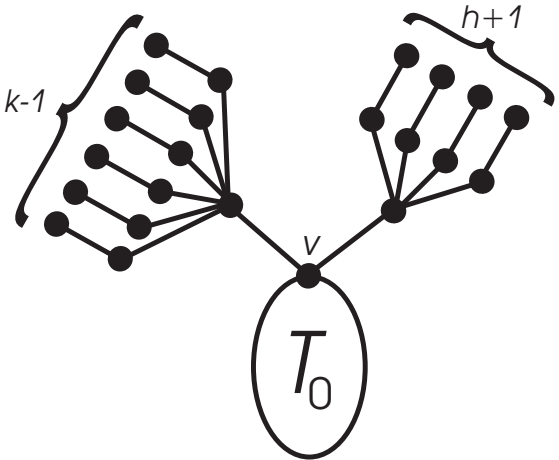

Y

Fig. 4. The trees considered in Theorem 4.

Theorem 4. The characteristic polynomials of the trees $T_{0}, X$, and $Y$ are related as:

$$
\phi(Y, \lambda)-\phi(X, \lambda)=(k-h+1) \lambda^{2}\left(\lambda^{2}-1\right)^{k+h-2} \phi\left(T_{0}, \lambda\right) .
$$

Proof. Applying Eq. (5) to the edges connecting the branches attached to $T_{0}$ we have

$$
\begin{aligned}
\phi(X, \lambda) & =\phi\left(T_{0}, \lambda\right) \phi\left(B_{k}, \lambda\right) \phi\left(B_{h}, \lambda\right)-\phi\left(T_{0}-v, \lambda\right) \phi\left(B_{h}, \lambda\right)\left(\lambda^{2}-1\right)^{k} \\
& -\phi\left(T_{0}-v, \lambda\right) \phi\left(B_{k}, \lambda\right)\left(\lambda^{2}-1\right)^{h} \\
\phi(Y, \lambda) & =\phi\left(T_{0}, \lambda\right) \phi\left(B_{k-1}, \lambda\right) \phi\left(B_{h+1}, \lambda\right)-\phi\left(T_{0}-v, \lambda\right) \phi\left(B_{h+1}, \lambda\right)\left(\lambda^{2}-1\right)^{k-1} \\
& -\phi\left(T_{0}-v, \lambda\right) \phi\left(B_{k-1}, \lambda\right)\left(\lambda^{2}-1\right)^{h+1} .
\end{aligned}
$$

Substitute into Eqs. (8) and (9) the expressions for the characteristic polynomial of $B_{k}$, Eq. (6), and the analogous formulas:

$$
\begin{aligned}
\phi\left(B_{h}, \lambda\right) & =\lambda\left(\lambda^{2}-1\right)^{h-1}\left(\lambda^{2}-h-1\right) \\
\phi\left(B_{k-1}, \lambda\right) & =\lambda\left(\lambda^{2}-1\right)^{k-2}\left(\lambda^{2}-k\right) \\
\phi\left(B_{h+1}, \lambda\right) & =\lambda\left(\lambda^{2}-1\right)^{h}\left(\lambda^{2}-h-2\right)
\end{aligned}
$$

and then calculate the difference between (9) and (8). After a lengthy but elementary calculation, in which most terms cancel out, we arrive at the identity (7).

According to formula (1), the term $\lambda^{2}\left(\lambda^{2}-1\right)^{k+h-2} \phi\left(T_{0}, \lambda\right)$ in Eq. (7) is in fact the characteristic polynomial of the graph consisting of a copy of $T_{0}$, of $k+h-2$ copies of $P_{2}$, and of two isolated vertices $P_{1}$. Denote this graph by $F$, and notice that it has $n\left(T_{0}\right)+2(k+$ 
$h-2)+2=n\left(T_{0}\right)+2(k+h+1)-4$ vertices. Thus, bearing in mind formula (1), we can rewrite Eq. (7) as

$$
\phi(Y, \lambda)-\phi(X, \lambda)=\phi(F, \lambda)
$$

where the order of the graph $F$ is by four smaller than the orders of $X$ and $Y$.

\section{Main results}

The trees $X$ and $Y$ considered in the previous section may be viewed as Kragujevac trees in which $v$ is the central vertex, and all other branches (if any) are same in both $X$ and $Y$. Then the transformation $X \rightarrow Y$ does not change either the order or the degree of these Kragujevac trees. Because of the requirement $k-2 \geq h$, in the transformation $X \rightarrow Y$, a larger branch is diminished and a smaller branch is increased. In other words, the difference in size between these two branches is reduced.

Now, if $\phi(X, \lambda)$ and $\phi(Y, \lambda)$ are polynomials of degree $n$, then by Eqs. (7) and (10), $\lambda^{2}\left(\lambda^{2}-1\right)^{k+h-2} \phi\left(T_{0}, \lambda\right) \equiv \phi(F, \lambda)$ is a polynomial of degree $n-4$. Since $F$ is acyclic, in view of identity (2),

$$
m(Y, k)=m(X, k)+(k-h+1) m(F, k-2)
$$

Since the multiplier $k-h+1$ is positive valued, we conclude that

$$
m(Y, k) \geq m(X, k)
$$

holds for all $k \geq 1$. Thus, Lemma 3 is applicable and we arrive at:

Lemma 5. If $X$ and $Y$ are Kragujevac trees of equal order and equal degree, and if their structure differs as indicated in Fig. 4, then provided $k-2 \geq h \geq 2$, the energy of $Y$ is greater than the energy of $X$.

Continuing the argument used to deduce Lemma 5, and repeating the transformations of the type $X \rightarrow Y$ as far as it is possible, we obtain our first main result:

Theorem 6. Among Kragujevac trees with fixed order $n$ and degree $d$, the species with maximal energy has either all branches isomorphic to $B_{k}$ if

$$
k=\frac{1}{2}\left(\frac{n-1}{d}-1\right)
$$

is an integer, or has branches isomorphic to $B_{k}$ and $B_{k+1}$ for

$$
k=\left\lfloor\frac{1}{2}\left(\frac{n-1}{d}-1\right)\right\rfloor .
$$

In other words, the branches of a maximal-energy Kragujevac tree are either equal or almost equal. 
Considering the transformation $Y \rightarrow X$ and repeating it as far as it is possible, we obtain our second main result:

Theorem 7. Among Kragujevac trees with fixed order $n$ and degree $d$, the species with minimal energy has all branches isomorphic to $B_{2}$ and a single branch isomorphic to $B_{k}$, where

$$
k=\frac{1}{2}[n-2-5(d-1)] .
$$

In other words, the minimal-energy Kragujevac tree has as many as possible branches of smallest size, i.e., $B_{2}$-branches.

\section{References}

[1] M. B. Ahmadi, S. A. Hosseini, P. Salehi Nowbandegani, On trees with minimal atom bond connectivity index, MATCH Commun. Math. Comput. Chem., Vol. 69, (2013), 559-563.

[2] M. B. Ahmadi, S. A. Hosseini, M. ZarrinderaKht, On large trees with minimal atombond connectivity index, MATCH Commun. Math. Comput. Chem., Vol. 69, (2013), 565-569.

[3] D. Cvetrović, M. Doob, H. Sachs, Spectra of Graphs - Theory and Application, Academic Press, New York, 1980.

[4] D. Cvet Ković, P. Rowlinson, S. Simić, An Introduction to the Theory of Graph Spectra, Cambridge Univ. Press, Cambridge, 2010.

[5] D. Dimitrov, Efficient computation of trees with minimal atom-bond connectivity index, Appl. Math. Comput., Vol. 224, (2013), 663-670.

[6] B. Furtula, A. Graovac, D. VukičEvić, Atom-bond connectivity index of trees, Discr. Appl. Math., Vol. 157, (2009), 2828-2835.

[7] B. Furtula, I. Gutman, M. Dehmer, On structure-sensitivity of degree-based topological indices, Appl. Math. Comput., Vol. 219, (2013), 8973-8978.

[8] B. Furtula, I. Gutman, M. Ivanović, D. VukičEvić, Computer search for trees with minimal ABC index, Appl. Math. Comput., Vol. 219, (2012), 767-772.

[9] I. Gutman, Acyclic systems with extremal Hückel $\pi$-electron energy, Theor. Chim. Acta, Vol. 45, (1977), 79-87.

[10] I. Gutman, The energy of a graph: Old and new results, Algebraic Combinatorics and Applications, (A. Betten, A. Kohnert, R. Laue, A. Wassermann, eds.), Springer, Berlin, 2001, 196-211.

[11] I. Gutman, Degree-based topological indices, Croat. Chem. Acta, Vol. 86, (2013), 351-361.

[12] I. Gutman, B. FurTUla, Trees with smallest atom-bond connectivity index, MATCH Commun. Math. Comput. Chem., Vol. 68, (2012), 131-136.

[13] I. Gutman, B. Furtula, M. B. Ahmadi, S. A. Hosseini, P. Salehi Nowbandegani, M. ZARRINDERAKHT, The ABC index conundrum, Filomat, Vol. 27, (2013), 1075-1083. 
[14] I. Gutman, B. Furtula, M. IVAnović, Notes on trees with minimal atom-bond connectivity index, MATCH Commun. Math. Comput. Chem., Vol. 67, (2012), 467-482.

[15] I. Gutman, H. Hosoya, On the calculation of the acyclic polynomial, Theor. Chim. Acta, Vol. 28, (1978), 279-286.

[16] I. Gutman, X. Li, J. Zhang, Graph energy, Analysis of Complex Networks. From Biology to Linguistics (M. Dehmer, F. Emmert-Streib, eds.), Wiley-VCH, Weinheim, 2009, 145-174.

[17] I. Gutman, J. Tošović, S. Radenković, S. Marković, On atom-bond connectivity index and its chemical applicability, Indian J. Chem., Vol. 51A, (2012), 690-694.

[18] F. HARARY, Graph Theory, Addison-Wesley, Reading, 1969.

[19] H. HosoyA, Graphical enumeration of the coefficients of the secular polynomials of the Hückel molecular orbitals, Theor. Chim. Acta, Vol. 25, (1972), 215-222.

[20] S. A. Hosseini, M. B. Ahmadi, I. Gutman, Kragujevac trees with minimal atom-bond connectivity index, MATCH Commun. Math. Comput. Chem., Vol. 71, (2014), 5-20.

[21] X. Li, Y. Shi, I. Gutman, Graph Energy, Springer, New York, 2012.

[22] W. Lin, X. LIN, T. GAO, X. WU, Proving a conjecture of Gutman concerning trees with minimal ABC index, MATCH Commun. Math. Comput. Chem., Vol. 69, (2013), 549-557. 